Research Article

\title{
High TXLNA Expression Predicts Favourable Outcome for Pancreatic Adenocarcinoma Patients
}

\author{
Shuangyu Lv $\mathbb{D}$, Guosen Zhang, Longxiang Xie, Zhongyi Yan $\mathbb{D}^{\mathbb{D}}$, Qiang Wang, \\ Yongqiang Li, Lu Zhang, Yali Han, Huimin Li, Yaowu Du, Yanjie Yang $\mathbb{D}$, \\ and Xiangqian Guo
}

Department of Preventive Medicine, Institute of Biomedical Informatics, Cell Signal Transduction Laboratory, Bioinformatics Center, Henan Provincial Engineering Center for Tumor Molecular Medicine, School of Basic Medical Sciences, Henan University, Kaifeng 475004, China

Correspondence should be addressed to Yanjie Yang; yangyj04@163.com and Xiangqian Guo; xqguo@henu.edu.cn

Received 20 October 2019; Accepted 30 January 2020; Published 25 February 2020

Academic Editor: Bilal Alatas

Copyright ( 2020 Shuangyu Lv et al. This is an open access article distributed under the Creative Commons Attribution License, which permits unrestricted use, distribution, and reproduction in any medium, provided the original work is properly cited.

TXLNA (taxilin alpha), a binding partner of the syntaxin family, was identified as a key factor in the coordination of intracellular vesicle trafficking and highly expressed in various tumor cells. However, the accurate relation between TXLNA and tumorigenesis and progression of pancreatic adenocarcinoma (PAAD) is still unclear. The present study was designed to examine the expression profile of TXLNA and explore its prognostic significance in PAAD patients and the possible molecular regulatory mechanism by analyzing a series of data from databases, including GEPIA, LOGpc, STRING, and GeneMANIA. The results indicate that TXLNA mRNA and protein were remarkably increased in PAAD tissues compared with normal pancreatic tissues. The high TXLNA expression was significantly correlated with superior overall survival (OS), disease-free interval (DFI), disease specific survival (DSS), and progression-free interval (PFI) for PAAD patients. In summary, high TXLNA expression could predict favourable OS, DFI, DSS, and PFI for PAAD patients, and it might be as potential prognostic biomarkers and targets for PAAD.

\section{Introduction}

Pancreatic cancer (PC) is one of the leading causes of cancer mortality, resulting in a substantial global burden [1]. It has become the third leading cause of cancer death in the USA, with a 5 -year survival rate of $8 \%$ [2]. In recent decades, the treatment of pancreatic cancer has been improved; however, the diagnostic and prognostic biomarkers for pancreatic cancer are still deficient due to its heterogeneous characteristics [3]. Up to now, carbohydrate antigen 19-9 (CA19-9) is the only available diagnostic biomarker for pancreatic adenocarcinoma (PAAD) approved by the Food and Drug Administration (FDA) in the U.S., but it had a limitation of poor sensitivity and specificity $[4,5]$. Therefore, it is urgent to search for novel biomarkers.

Taxilin alpha (TXLNA) was identified as a binding partner of the syntaxin family [6]. The TXLNA was also named interleukin 14 (IL-14), and its alternative gene name was designated as TXLNA or IL14 in the NCBI. The function of TXLNA was identified as a key factor in the coordination of intracellular vesicle trafficking [6]. The syntaxin family was composed of three members, including alpha $(\alpha)$-, beta $(\beta)$-, and gamma $(\gamma)$-taxilin. In addition, they share an extraordinarily long coiled-coil region homologous to that of Uso1, a yeast tethering factor homolog of p115 [7]. It was reported that the syntaxin family involved in the transport of the vesicles delivered to the plasma membrane $[6,7]$. As we know, the intracellular vesicle tranfficking is a primary transportation system in eukaryotic cells, and vesicle tranfficking participates in the multiple processes of cell proliferation. The abnormal proliferation indicates a high probability of tumor or cancer [8].

Recent studies demonstrate that the TXLNA protein had a high expression in various tumor cells and its expression indicates a close relationship with histological grade and proliferative activity in hepatocellular carcinoma, as well as 
the metastatic and invasive potential of renal cell carcinoma [8-11]. Sakakibara et al. showed that a strong expression of TXLNA was detected in proliferating neuroepithelial cells during embryonic development of the rat brain [12]. Horii et al. have found that the expression of TXLNA was correlated with cell proliferation in the adult murine gastrointestinal tract and during postnatal development [9].

The relationship between TXLNA and pancreatic cancer prognosis remains to be determined. The present study was designed to evaluate the expression profile of TXLNA and to investigate its prognostic significance for PAAD patients.

\section{Materials and Methods}

2.1. TXLNA Gene Expression Analysis. The TXLNA mRNA level in PAAD tissue and normal pancreatic tissue was examined by on-line analysis GEPIA (Gene Expression Profiling Interactive Analysis) (http://gepia.cancer-pku.cn). GEPIA is a web server which analyzes the RNA expression data of 9,736 tumors and 8,587 normal samples from TCGA and GTEx [13].

2.2. Prognosis Analysis. The relationship between the expression of TXLNA and survival terms, including overall survival (OS), disease-free interval (DFI), disease specific survival (DSS), and progression-free interval (PFI), in 542 PAAD patients was determined by the prognosis analysis web server LOGpc [14] (Long-term Outcome and Gene Expression Profiling Database of pan-cancers, http://bioinfo. henu.edu.cn/DatabaseList.jsp) using TCGA and ICGA_Seq as we previously reported [15]. This LOGpc tool encompasses 171 expression datasets and provides 13 types of survival terms for 27929 patients of 26 distinct malignancies.

2.3. Gene Interaction Network Construction. Search Tool for the Retrieval of Interacting Genes (STRING, version 11.0, available online: http://www.string-db.org/) is an opensource database, which was applied to predict proteinprotein interaction (PPI) networks of differentially expressed genes (DEGs) [16]. In addition, only the interactions with a combined score $>0.4$ were considered to have a statistically significant difference. The present new version contains 5090 organisms, 24584628 proteins, and 3123056667 total interactions. GeneMANIA (http:// genemania.org/) was utilized to conduct analysis of TXLNA correlated genes. GeneMANIA is a prediction server to search for other genes related to the targeted genes using analysis of functional association, including protein and genetic interactions, pathways, coexpression, colocalization, and protein domain similarity [17].

2.4. Statistical Analysis. Univariate and multivariate Cox regression models were utilized to assess the prognostic role of TXLNA in terms of OS, DFI, PFI, and DSS for PAAD patients using SPSS. Risk factors $(p<0.05)$ analyzed by univariate analysis were selected for multivariate Cox regression analysis. A value of $p<0.05$ was considered statistically significant.

\section{Results}

3.1. TXLNA Expression in PAAD Tissue Was Increased Compared with Normal Tissue. As shown in Figure 1(a), the GEPIA analysis based on TCGA and the GTEx data indicates that PAAD tissues exhibited a significantly increased TXLNA expression, compared with the normal tissue $(p<0.05)$. There was no significant difference of TXLNA expression between the TNM stages $(p=0.47$, Figure $1(\mathrm{~b}))$ and different neoplasm histologic grades $(p=0.267$, Figure S1).

3.2. High TXLNA Expression Was Correlated with Favourable OS, DFI, DSS, and PFI in PAAD Patients. The prognostic value of TXLNA was tested by KaplanMeier Plotter Analysis of LOGpc $[18,19]$. Analysis based on TCGA data in LOGpc showed that the high TXLNA expression group had favourable OS $(p=0.0175$; HR: $0.5519 ; \quad 95 \%$ CI: 0.338-0.9013; Figure 2(a)), DFI $(p=0.0149$; HR: 0.4971; 95\% CI: 0.2832-0.8725; Figure 2(b)), DSS $(p=0.05$; HR: 0.5549; 95\% CI: 0.3079-1.0001; Figure 2(c)), and PFI $(p=0.0153 ; \quad H R: \quad 0.5515 ; \quad 95 \% \quad$ CI: $0.3409-0.8921$; Figure 2(d)) for PAAD patients. Meanwhile, analysis results from ICGA_Seq data demonstrated that the increased TXLNA expression group had favourable OS $(p=0.0015$; HR: 0.5786 ; 95\% CI: 0.4128-0.8109; Figure 2(e)) and favourable DFI $(p=0.0448$; HR: $0.6577 ; 95 \%$ CI: 0.4367-0.9904; Figure 2(f)) for PAAD patients. These results indicate that high TXLNA expression was significantly correlated with favourable OS, DFI, DSS, and PFI in PAAD patients.

3.3. Significant Correlation between the Increased TXLNA Expression and Favourable OS in PAAD Patients Was Restricted to Female Patients or Patients with Lymph Nodes, NoSmoking History, or Alcohol History. To examine whether the correlation was influenced by the different clinical characteristics of PAAD patients, such as gender, lymph nodes, and smoking or alcohol, each character was used to divide PAAD patients into two subgroups (male and female, lymph nodes and no-lymph nodes, smoking and nosmoking, and alcohol and no-alcohol) for analysis. The high TXLNA expression group had favourable OS in female PAAD patients $(p=0.0047$; HR: $0.3509 ; 95 \%$ CI: $0.1698-0.7248$; Figure 3(a)), but not in male PAAD patients ( $p=0.5052$; HR: $0.7952 ; \quad 95 \%$ CI: $0.4053-1.5602$; Figure $3(\mathrm{~b})$ ). The increased TXLNA expression group had favourable $\mathrm{OS}$ in PAAD patients with lymph nodes $(p=0.0284$; HR: $0.5773 ; \quad 95 \% \quad$ CI: $0.3531-0.9437$; Figure 3(c)), but not in PAAD patients with no-lymph nodes $(p=0.9996 ;$ Figure $3(\mathrm{~d}))$. The high TXLNA expression group had favourable OS in PAAD patients with nosmoking history $(p=0.0101$; HR: $0.366 ; 95 \%$ CI: 0.1701-0.7872; Figure 3(e)), but not in PAAD patients with smoking history $(p=0.3236$; HR: $0.6551 ; 95 \%$ CI: 0.2828-1.5174; Figure 3(f)). The increased TXLNA expression group had favourable OS in PAAD patients with alcohol history ( $p=0.0454$; HR: 0.5088 ; 95\% CI: $0.2625-0.9861$; 


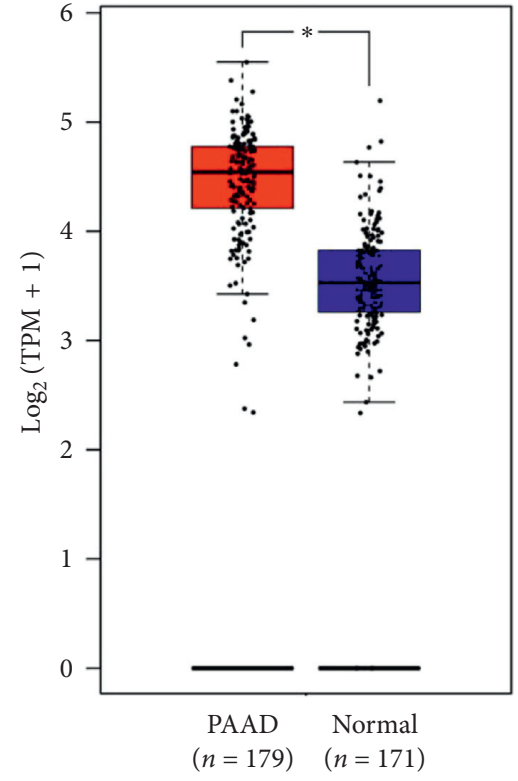

(a)

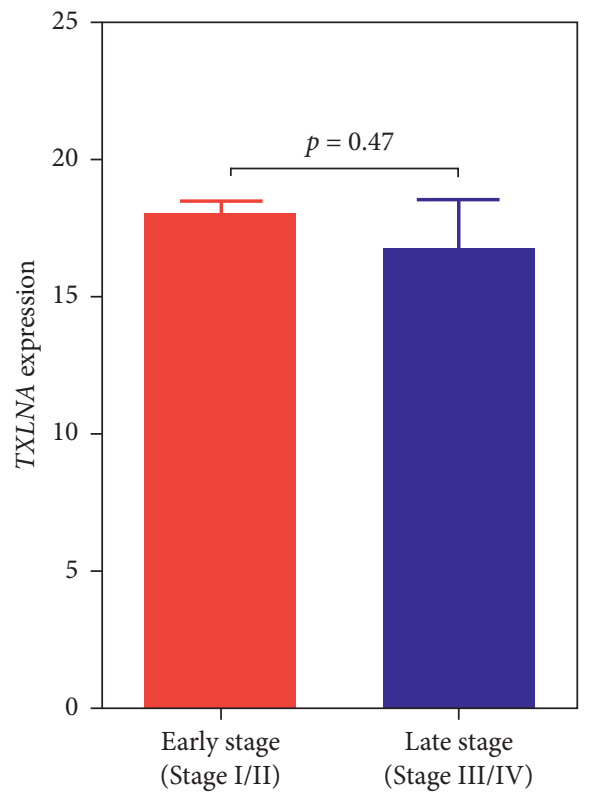

(b)

Figure 1: The TXLNA gene expression in pancreatic adenocarcinoma (PAAD) tissue and normal pancreas tissue. (a) The TXLNA expression in tumor tissue and normal tissue was analyzed using Gene Expression Profiling Interactive Analysis (GEPIA). (b) The TXLNA expression at early and late clinical stages of PAAD cases was analyzed based on the TCGA dataset. TPM, transcripts per million reads.

Figure $3(\mathrm{~g}))$, but not in PAAD patients with no-alcohol history $(p=0.1046$; HR: 0.5048 ; $95 \%$ CI: $0.2211-1.1525$; Figure 3(h)). These results demonstrated that high TXLNA expression indicated a favourable OS in female patients or patients with lymph nodes, no-smoking history, or alcohol history.

3.4. TXLNA Expression Was an Independent Prognostic Biomarker for Favourable OS, DFI, and PFI in PAAD. Univariate analysis demonstrated that the lymph nodes and increased TXLNA expression correlated with favourable OS, DFI, PFI, and DSS for PAAD (Table 1). Multivariate analysis indicated that high TXLNA expression was an independent prognostic indicator in terms of OS (HR: 1.641; 95\% CI: $1.003-2.685 ; p=0.048)$, DFI (HR: $1.848 ; 95 \% \quad \mathrm{CI}$ : $1.051-3.250 ; p=0.033$ ), and PFI (HR: $1.668 ; 95 \% \mathrm{CI}$ : 1.029-2.702; $p=0.038$; Table 1).

3.5. Construction of Protein-Protein and Gene-Gene Interaction Network. To explore the potentially interacted proteins with TXLNA, the PPI network was constructed by STRING. After removing unconnected nodes, the PPI network containing 41 nodes and 310 edges was established (Figure 4(a)). To further investigate the hub proteins in the network, the top 10 hub proteins were retrieved: STX3, STX4, STX1A, STXBP2, VAMP2, SNAP25, TXLNG, DDX17, TXLNB, and QARS (Figure 4(b)). Gene-gene interaction analysis using GeneMANIA showed 20 correlated genes with TXLNA, such as TXLNB, TXLNG, NACA, TTC27, IKBKG, TXNDC12, WIPI2, BRCA1, VAMP2, and KIF3C (Figure 4(c)).

\section{Discussion}

Pancreatic cancer is a highly heterogeneous disease with a low overall survival rate $[2,3]$. Therefore, it is essential to discover effective tumor biomarkers to improve the clinical diagnosis, prognosis, and treatment. Recently, a variety of prognostic markers for pancreatic cancer are reported. Li et al. had found that the combination of SRPX2 and RAB31 were independent prognostic factors that associated with OS and DFS of pancreatic cancer [20]. Chen et al. reported that three hypomethylated genes (SULT1E1, IGF2BP3, and MAP4K4) were associated with poor overall survival of pancreatic cancer patients [21]. Pang et al. have indicated that IL22RA1, BCL2L1, STAT1, $M Y C$, and STAT2 involved in the Jak-STAT signaling pathway may be significantly associated with prognosis of pancreatic cancer [22].

Recent studies indicate that TXLNA was involved in inflammation, endocrine, and respiratory diseases. IL-14, a cytokine, was identified as high molecular weight B-cell growth factor [23]. Its mRNA transcript level was increased in vitro in human $\mathrm{T}$ cells responding to alloantigen stimulation [24]. The IL-14, IL-17, and TNF- $\alpha$ level in whole lung homogenates were upregulated in RAGE transgenic mice [25]. Helen Kemp et al. had demonstrated that the thyroid follicular cells in the thyroid had the ability to express IL-14 mRNA [26]. Nevertheless, the role of TXLNA on pancreatic cancer remains unknown.

For PAAD patients, prognostic factors could help to guide the personalized treatments. The correlation of TXLNA expression and prognostic value was analyzed using LOGpc. We discovered that increased TXLNA expression was significantly correlated with favourable OS, DFI, PFI, 

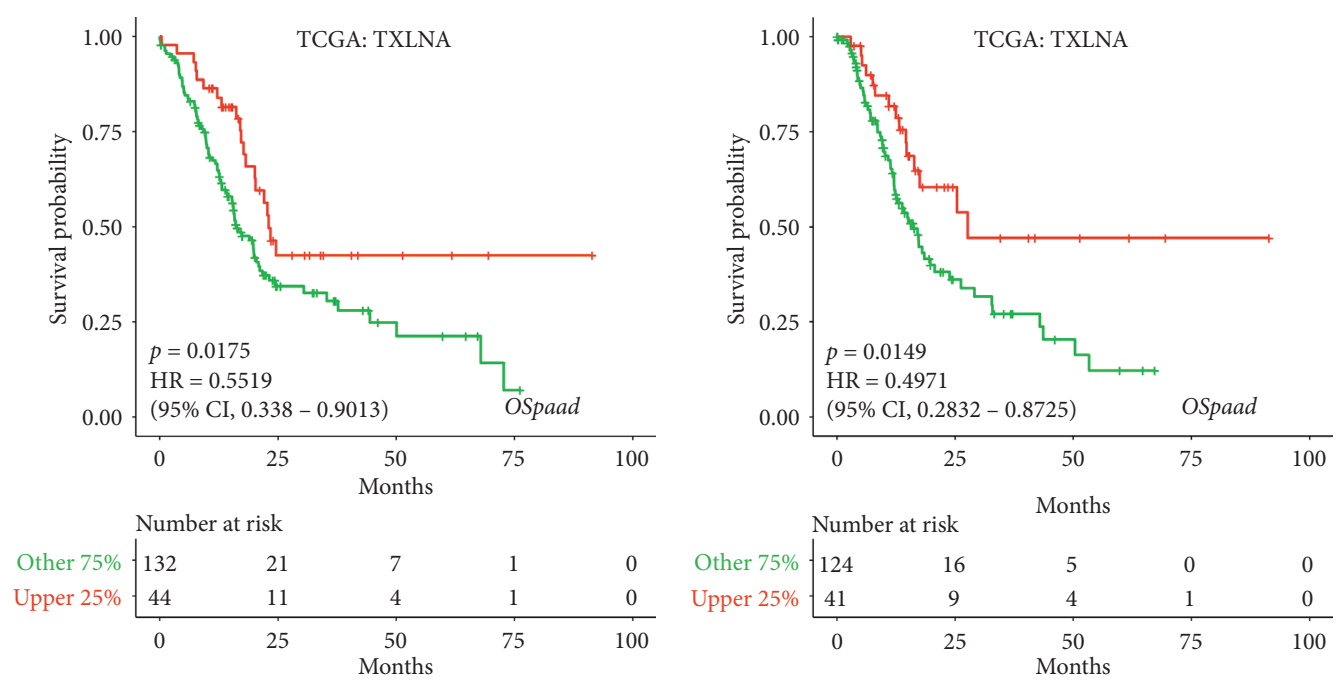

(a)

(b)
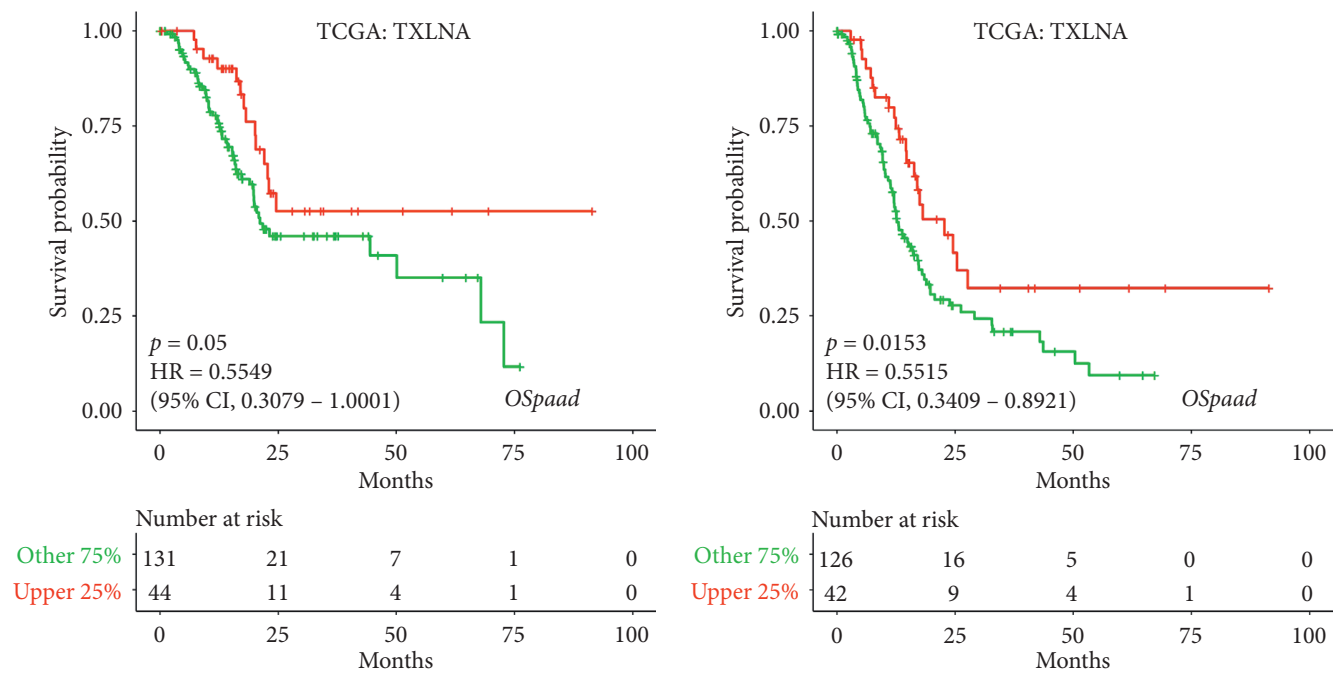

(c)

(d)
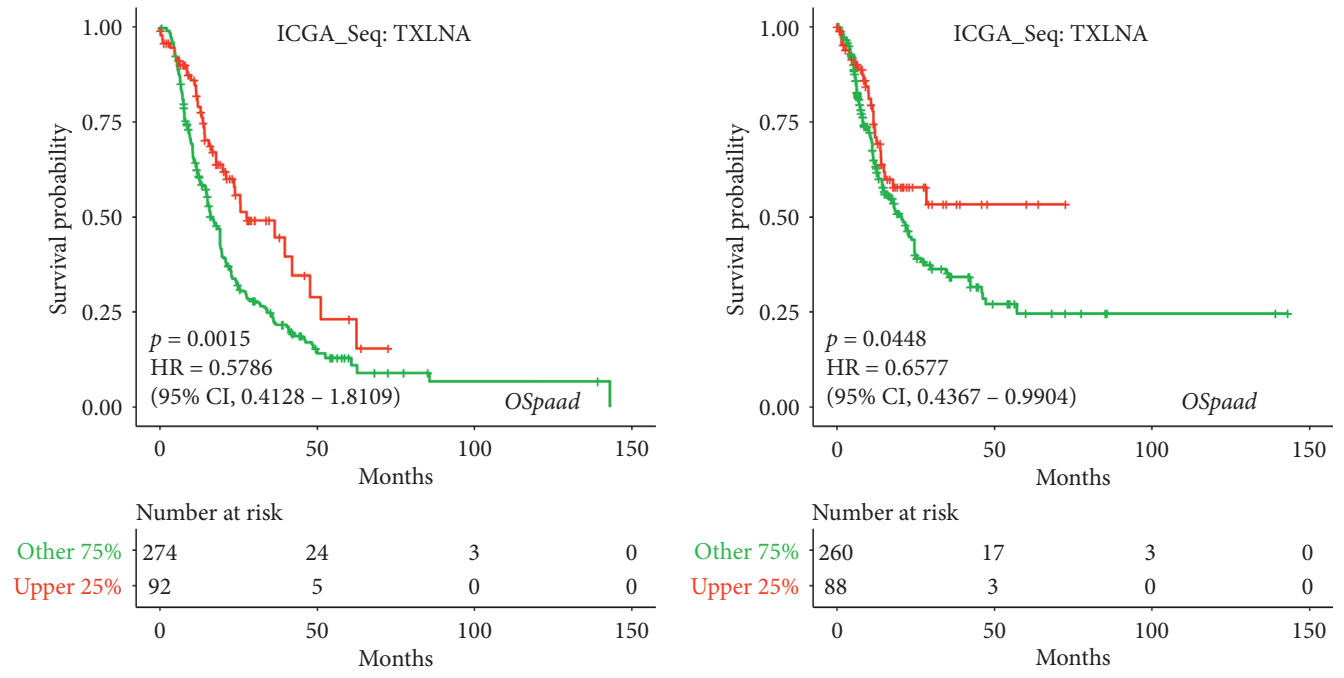

(e)

(f)

FIgURE 2: Kaplan-Meier curves of overall survival (OS) (a), disease-free interval (DFI) (b), disease specific survival (DSS) (c), and progression-free interval (PFI) (d) from TCGA data in PAAD. Kaplan-Meier curves of overall survival (OS) (e) and disease-free interval (DFI) (f) from ICGA data in PAAD. 

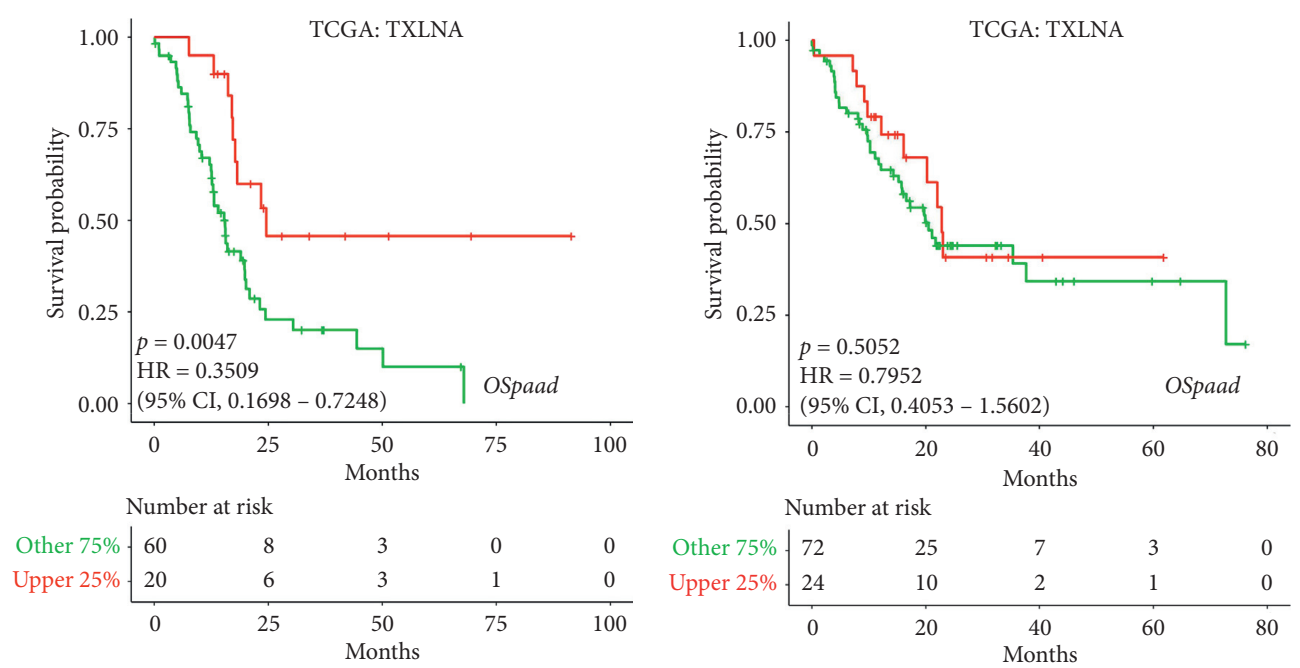

(a)
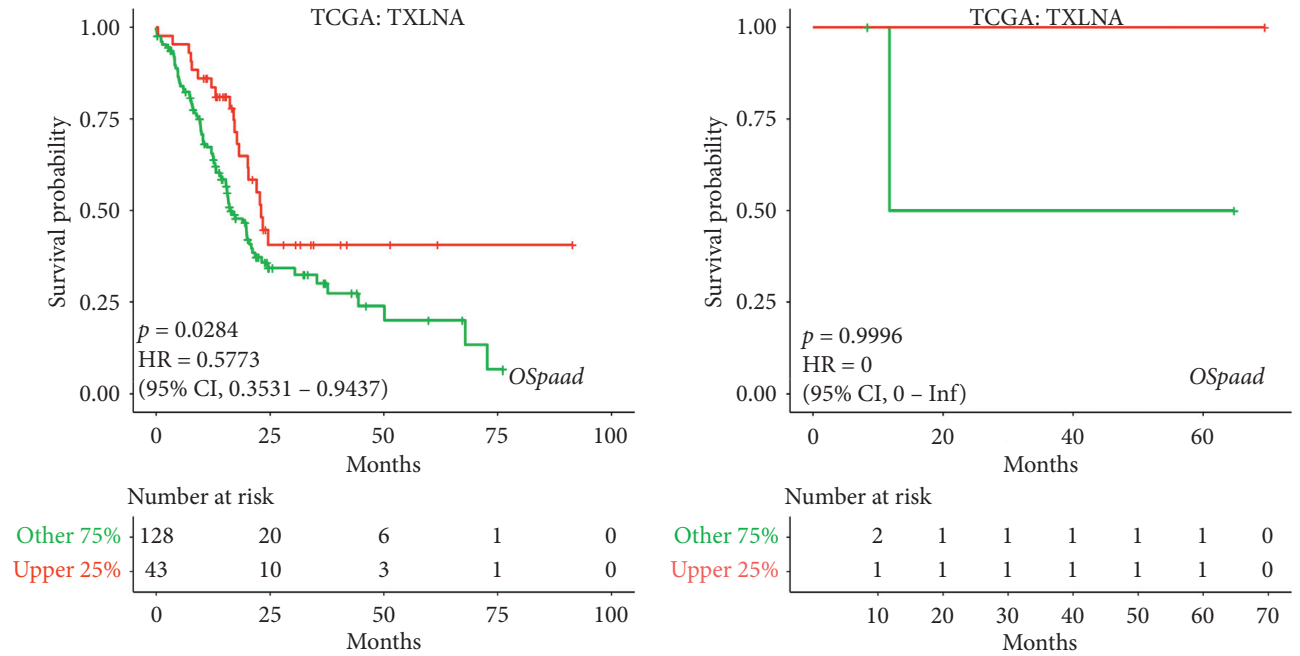

(c)

(d)
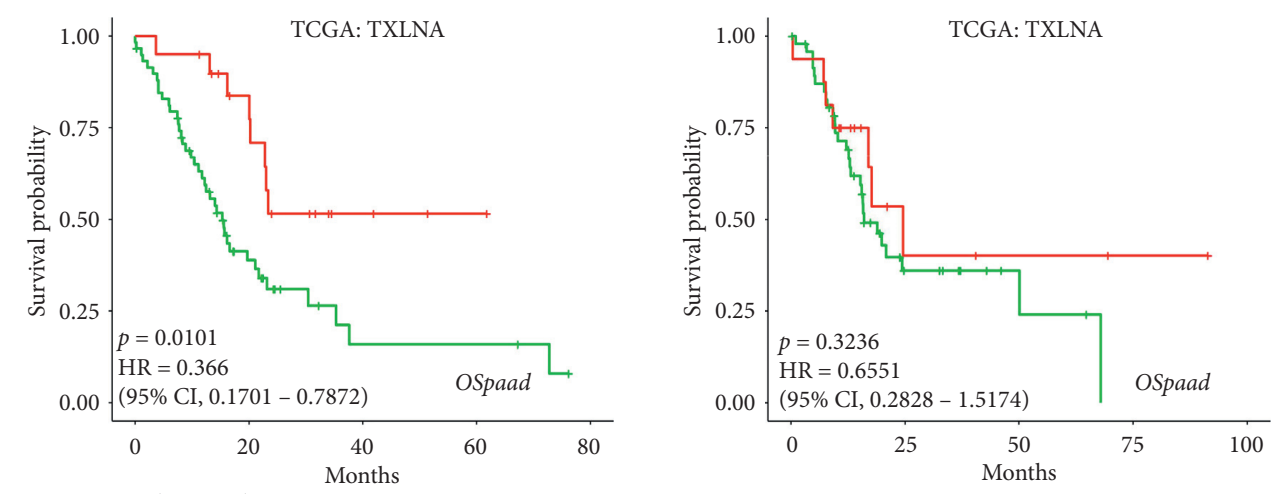

Number at risk

Other 75\% 5 Upper 25\% \begin{tabular}{ccccc}
59 & 16 & 3 & 3 & 0 \\
20 & 13 & 3 & 1 & 0 \\
\cline { 2 - 5 } & 20 & 40 & 60 & 80 \\
& & Months & &
\end{tabular}

(e)

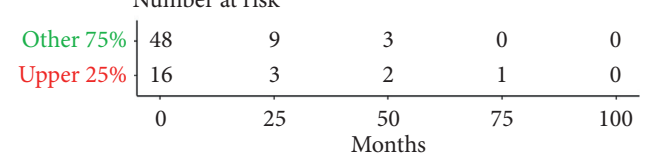

(f)

Figure 3: Continued. 

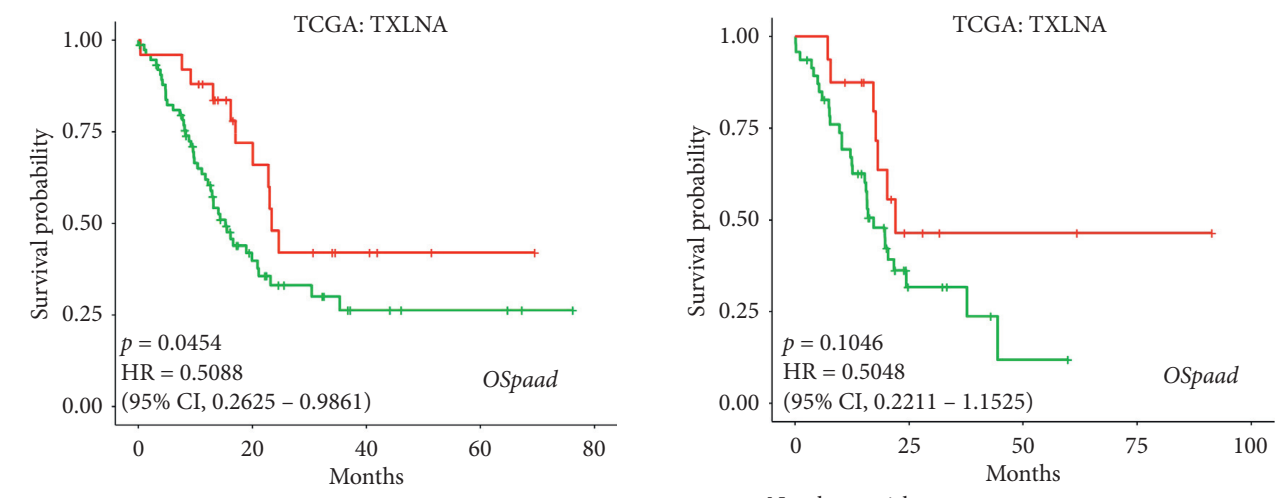

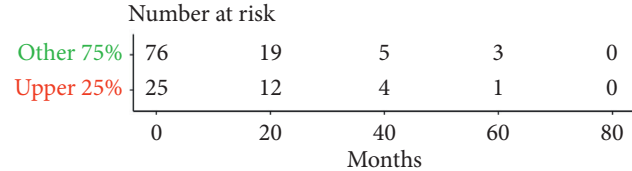

(g)

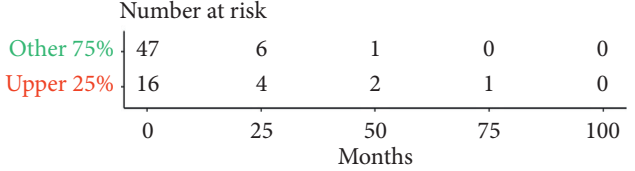

(h)

Figure 3: Kaplan-Meier curves of overall survival (OS) in female (a), male (b), lymph nodes (c), no-lymph nodes (d), no-smoking (e), smoking (f), alcohol (g), and no-alcohol (h) from TCGA data in PAAD.

TABle 1: Univariate and multivariate analysis of OS, DFI, PFI, and DSS in patients with PAAD in the TCGA.

\begin{tabular}{|c|c|c|c|c|c|c|c|c|}
\hline \multirow{2}{*}{ Parameters } & \multicolumn{4}{|c|}{ Univariate analysis } & \multicolumn{4}{|c|}{ Multivariate analysis } \\
\hline & $p$ & HR & $95 \%($ & apper) & $p$ & HR & $95 \%($ & pper) \\
\hline \multicolumn{9}{|l|}{ OS } \\
\hline $\begin{array}{l}\text { Age } \\
>65 \text { vs. } \leq 65\end{array}$ & 0.141 & 0.752 & 0.515 & 1.099 & & & & \\
\hline Female vs. male & 0.228 & 0.793 & 0.545 & 1.156 & & & & \\
\hline $\begin{array}{l}\text { Smoking history } \\
2 / 3 / 4 / 5 \text { vs. } 1\end{array}$ & 0.732 & 1.076 & 0.706 & 1.641 & & & & \\
\hline $\begin{array}{l}\text { Alcohol history } \\
\text { Yes vs. no }\end{array}$ & 0.878 & 1.033 & 0.685 & 1.558 & & & & \\
\hline $\begin{array}{l}\text { Lymph node } \\
\text { yes vs. no }\end{array}$ & 0.001 & 2.236 & 1.373 & 3.641 & 0.004 & 0.477 & 0.288 & 0.788 \\
\hline $\begin{array}{l}\text { Clinical stage } \\
\text { III/IV vs. II/I }\end{array}$ & 0.807 & 0.894 & 0.363 & 2.202 & & & & \\
\hline $\begin{array}{l}\text { TXLNA expression } \\
\text { High vs. low } \\
\text { DFI }\end{array}$ & 0.018 & 1.811 & 1.109 & 2.958 & 0.048 & 1.641 & 1.003 & 2.685 \\
\hline $\begin{array}{l}\text { Age } \\
>65 \text { vs. } \leq 65\end{array}$ & 0.287 & 0.787 & 0.507 & 1.222 & & & & \\
\hline Female vs. male & 0.661 & 0.908 & 0.591 & 1.396 & & & & \\
\hline $\begin{array}{l}\text { Smoking history } \\
2 / 3 / 4 / 5 \text { vs. } 1\end{array}$ & 0.502 & 1.173 & 0.736 & 1.868 & & & & \\
\hline $\begin{array}{l}\text { Alcohol history } \\
\text { Yes vs. no }\end{array}$ & 0.382 & 0.810 & 0.504 & 1.300 & & & & \\
\hline $\begin{array}{l}\text { Lymph node } \\
\text { Yes vs. no }\end{array}$ & 0.033 & 0.585 & 0.358 & 0.957 & 0.048 & 0.601 & 0.363 & 0.996 \\
\hline $\begin{array}{l}\text { Clinical stage } \\
\text { III/IV vs. II/I }\end{array}$ & 0.328 & 0.561 & 0.176 & 1.786 & & & & \\
\hline $\begin{array}{l}\text { TXLNA expression } \\
\text { High vs. low } \\
\text { PFI }\end{array}$ & 0.016 & 1.999 & 1.139 & 3.509 & 0.033 & 1.848 & 1.051 & 3.250 \\
\hline $\begin{array}{l}\text { Age } \\
>65 \text { vs. } \leq 65\end{array}$ & 0.159 & 1.318 & 0.897 & 1.937 & & & & \\
\hline Female vs. male & 0.815 & 0.956 & 0.656 & 1.394 & & & & \\
\hline $\begin{array}{l}\text { Smoking history } \\
2 / 3 / 4 / 5 \text { vs. } 1\end{array}$ & 0.786 & 1.059 & 0.7 & 1.602 & & & & \\
\hline
\end{tabular}


TABle 1: Continued.

\begin{tabular}{|c|c|c|c|c|c|c|c|c|}
\hline \multirow{3}{*}{$\begin{array}{l}\text { Parameters } \\
\text { Alcohol history } \\
\text { Yes vs. no }\end{array}$} & \multicolumn{4}{|c|}{ Univariate analysis } & \multicolumn{4}{|c|}{ Multivariate analysis } \\
\hline & \multirow{2}{*}{$\frac{p}{0.63}$} & \multirow{2}{*}{$\frac{\mathrm{HR}}{0.902}$} & \multicolumn{2}{|c|}{ 95\% (lower/upper) } & \multirow[t]{2}{*}{$p$} & \multirow[t]{2}{*}{ HR } & \multicolumn{2}{|c|}{ 95\% (lower/upper) } \\
\hline & & & 0.593 & 1.372 & & & & \\
\hline $\begin{array}{l}\text { Lymph node } \\
\text { Yes vs. no }\end{array}$ & 0.004 & 0.524 & 0.336 & 0.819 & 0.012 & 0.558 & 0.354 & 0.881 \\
\hline $\begin{array}{l}\text { Clinical stage } \\
\text { III/IV vs. II/I }\end{array}$ & 0.922 & 1.039 & 0.481 & 2.247 & & & & \\
\hline $\begin{array}{l}\text { TXLNA expression } \\
\text { High vs. Low } \\
\text { DSS }\end{array}$ & 0.017 & 1.8 & 1.113 & 2.912 & 0.038 & 1.668 & 1.029 & 2.702 \\
\hline $\begin{array}{l}\text { Age } \\
>65 \text { vs. } \leq 65\end{array}$ & 0.640 & 1.115 & 0.706 & 1.762 & & & & \\
\hline Female vs. male & 0.500 & 0.855 & 0.543 & 1.347 & & & & \\
\hline $\begin{array}{l}\text { Smoking history } \\
2 / 3 / 4 / 5 \text { vs. } 1\end{array}$ & 0.463 & 0.827 & 0.498 & 1.373 & & & & \\
\hline $\begin{array}{l}\text { Alcohol history } \\
\text { Yes vs. no }\end{array}$ & 0.493 & 0.835 & 0.498 & 1.398 & & & & \\
\hline $\begin{array}{l}\text { Lymph node } \\
\text { Yes vs. no }\end{array}$ & 0.004 & 0.411 & 0.226 & 0.750 & 0.01 & 0.438 & 0.234 & 0.82 \\
\hline $\begin{array}{l}\text { Clinical stage } \\
\text { III/IV vs. II/I }\end{array}$ & 0.561 & 1.311 & 0.526 & 3.270 & & & & \\
\hline $\begin{array}{l}\text { TXLNA expression } \\
\text { High vs. low }\end{array}$ & 0.050 & 1.802 & 1.000 & 3.247 & 0.103 & 1.635 & 0.906 & 2.951 \\
\hline
\end{tabular}

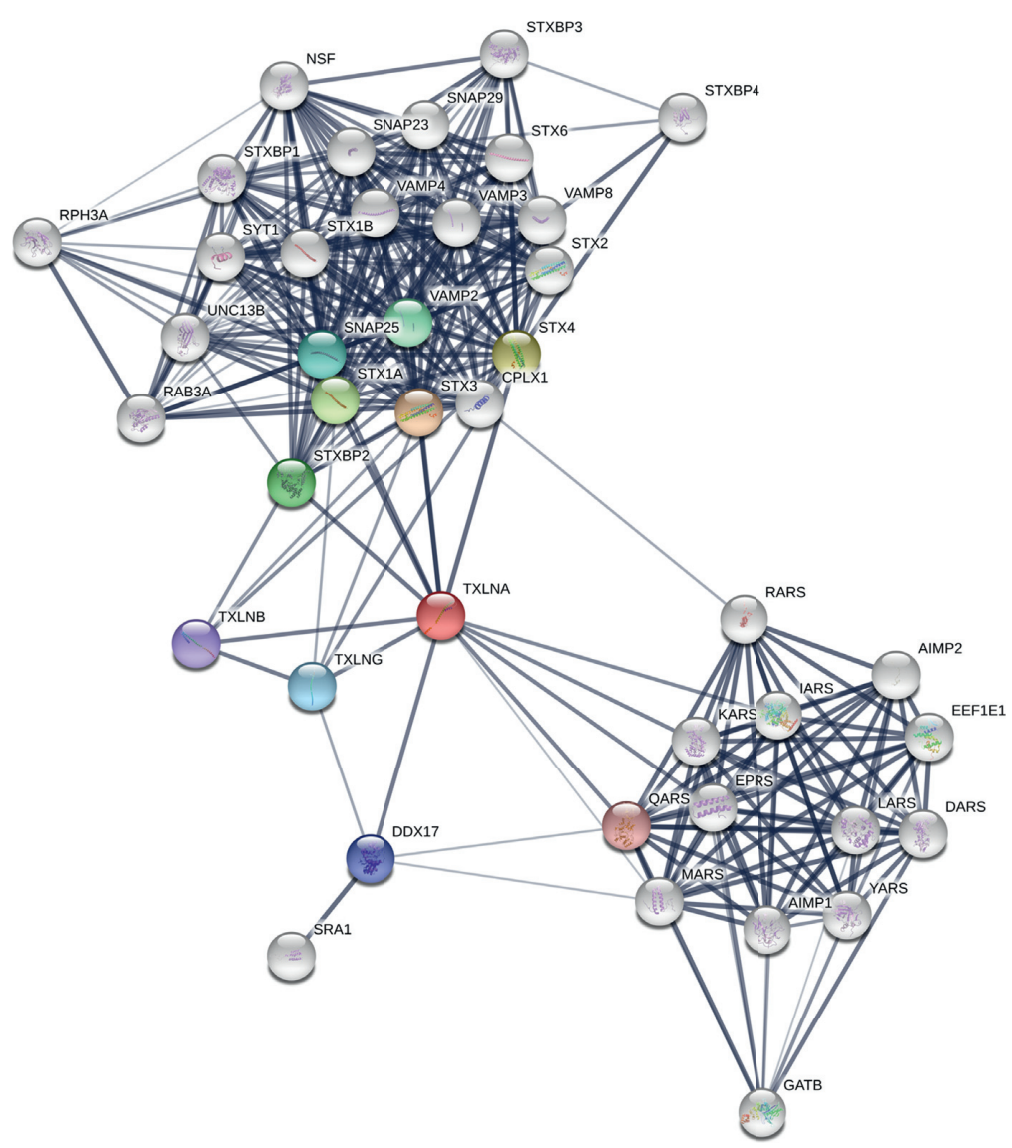

(a)

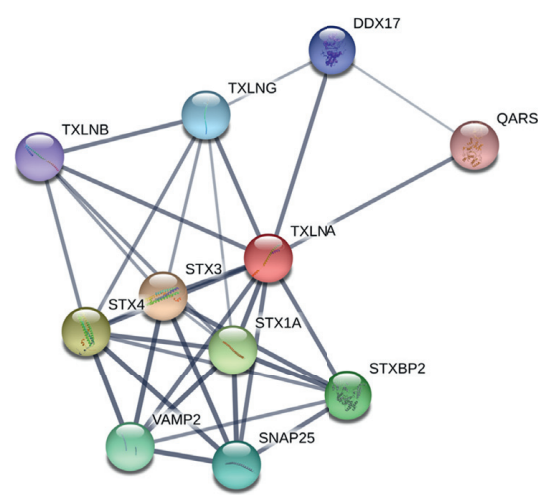

Figure 4: Continued.

(b) 


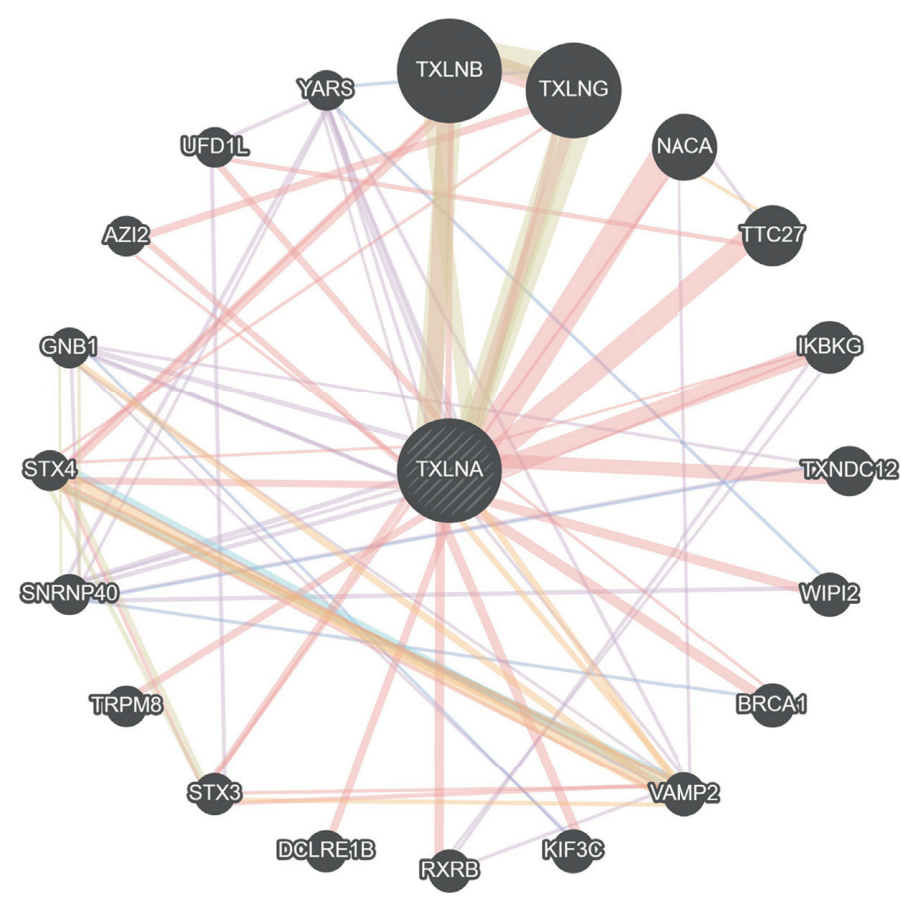

(c)

FIGURE 4: Interaction analysis of TXLNA at the gene and protein levels. (a) The PPI network constructed with STRING contained 41 nodes and 310 edges. (b) The PPI network of 10 hub proteins (34 edges) was extracted from A. The line thickness indicates the strength of data support. (c) Gene-gene interaction network for TXLNA was analyzed using GeneMANIA prediction server (http://genemania.org/).

and DSS in PAAD patients in TCGA data. Analysis from ICGA_Seq data also indicated that the upregulated TXLNA expression was significantly correlated with favourable OS and DFI. In addition, further analysis suggested that the correlation of high TXLNA expression and favourable OS was influenced by gender, smoking, alcohol, and lymph history in patients with PAAD. Gender was a key factor for prognostic analysis. Previous studies indicated that gender had a significant influence on the outcome of prognostic analysis, such as disease-free survival, and distant metastasis [27-29]. Our study showed that the high TXLNA expression group exhibited favourable OS in female PAAD patients, but not in male. The gender differences in the prognosis of PAAD could be explained by genetic variants affected by the hormonal environment. It suggests that high TXLNA expression was suitable for prognosis of female (not male) PAAD patients. Multivariate analysis showed that high TXLNA expression was an independent prognostic indicator in terms of OS, DFI, and PFI in PAAD patient.

In conclusion, TXLNA has a high expression in PAAD tissue compared with normal tissue, and its upregulated expression might serve as an independent prognostic indicator of OS, DFI, DSS, and PFI in PAAD patients. Further experimental studies are needed to fully understand the association between TXLNA expression and prognosis of PAAD patients.

\section{Data Availability}

The data used to support the findings of this study are available from the corresponding author upon request.

\section{Conflicts of Interest}

The authors declare that they have no conflicts of interest.

\section{Authors' Contributions}

$X G$ and YY developed the idea and designed the research. SL, GZ, and LX collected and analyzed the data. SL, ZY, and QW wrote the draft of the manuscript. YL, LZ, YH, $\mathrm{HL}$, and YD contributed to revise the writing. All authors read and approved the submitted version. Shuangyu Lv and Guosen Zhang authors have contributed equally to this work.

\section{Acknowledgments}

This work was supported by the National Natural Science Foundation of China (no. 81602362, 81600974, and 81971280), the Key Science and Technology Program of Henan Province in China (no. 162102310391 and 192102310080), the supporting grants of Henan University (no. 2015YBZR048 and B2015151), the Research Program for Young Talent of Henan University School of Medicine (Grant no. 2019018), the Program for Innovative Talents of Science and Technology in Henan Province (no. 18HASTIT048), the Yellow River Scholar Program (no. H2016012), the Kaifeng Science and Technology Major Project (no. 18ZD008), and Key Science and Technology Program of Kaifeng City (no. 1803034 and 1903019). 


\section{Supplementary Materials}

Supplementary Figure 1: the TXLNA gene expression in PAAD patients with different grades. The histogram indicates the expression level of the TXLNA gene at different neoplasm histologic grades (G1, G2, G3, G4, and GX) in patients with PAAD in the TCGA. The values were expressed as the mean \pm S.E.M. A one-way analysis of variance (ANOVA) followed by the Tukey's test for post hoc comparisons was used for the statistical evaluation. (Supplementary Materials)

\section{References}

[1] J. Ferlay, I. Soerjomataram, R. Dikshit et al., "Cancer incidence and mortality worldwide: sources, methods and major patterns in GLOBOCAN 2012," International Journal of Cancer, vol. 136, no. 5, pp. E359-E386, 2015.

[2] R. L. Siegel, K. D. Miller, and A. Jemal, "Cancer statistics, 2018," CA: A Cancer Journal for Clinicians, vol. 68, no. 1, pp. 7-30, 2018.

[3] N. Samuel and T. J. Hudson, "The molecular and cellular heterogeneity of pancreatic ductal adenocarcinoma," Nature Reviews Gastroenterology \& Hepatology, vol. 9, no. 2, pp. 77-87, 2012.

[4] K. S. Goonetilleke and A. K. Siriwardena, "Systematic review of carbohydrate antigen (CA 19-9) as a biochemical marker in the diagnosis of pancreatic cancer," European Journal of Surgical Oncology (EJSO), vol. 33, no. 3, pp. 266-270, 2007.

[5] Y. Zhang, J. Yang, H. Li, Y. Wu, H. Zhang, and W. Chen, "Tumor markers CA19-9, CA242 and CEA in the diagnosis of pancreatic cancer: a meta-analysis," International Journal of Clinical and Experimental Medicine, vol. 8, no. 7, pp. 11683-11691, 2015.

[6] S. Nogami, S. Satoh, M. Nakano et al., "Taxilin; a novel syntaxin-binding protein that is involved in $\mathrm{Ca} 2+$-dependent exocytosis in neuroendocrine cells," Genes to Cells, vol. 8, no. 1, pp. 17-28, 2003.

[7] S. Nogami, S. Satoh, S. Tanaka-Nakadate et al., "Identification and characterization of taxilin isoforms," Biochemical and Biophysical Research Communications, vol. 319, no. 3, pp. 936-943, 2004.

[8] T. Mashidori, H. Shirataki, T. Kamai, F. Nakamura, and K.-I. Yoshida, "Increased alpha-taxilin protein expression is associated with the metastatic and invasive potential of renal cell cancer," Biomedical Research, vol. 32, no. 2, pp. 103-110, 2011.

[9] Y. Horii, H. Sakane, S. Nogami, N. Ohtomo, T. Tomiya, and H. Shirataki, "Expression of $\alpha$-taxilin in the murine gastrointestinal tract: potential implication in cell proliferation," Histochemistry and Cell Biology, vol. 141, no. 2, pp. 165-180, 2014.

[10] S. M. Oba-Shinjo, M. H. Bengtson, S. M. B. Winnischofer et al., "Identification of novel differentially expressed genes in human astrocytomas by cDNA representational difference analysis," Molecular Brain Research, vol. 140, no. 1-2, pp. 25-33, 2005.

[11] N. Ohtomo, T. Tomiya, Y. Tanoue et al., "Expression of alphataxilin in hepatocellular carcinoma correlates with growth activity and malignant potential of the tumor," International Journal of Oncology, vol. 37, no. 6, pp. 1417-1423, 2010.

[12] S.-I. Sakakibara, K. Nakadate, S. Tanaka-Nakadate et al., "Developmental and spatial expression pattern of $\alpha$-Taxilin in the rat central nervous system," The Journal of Comparative Neurology, vol. 511, no. 1, pp. 65-80, 2008.

[13] Z. Tang, C. Li, B. Kang, G. Gao, C. Li, and Z. Zhang, "GEPIA: a web server for cancer and normal gene expression profiling and interactive analyses," Nucleic Acids Research, vol. 45, no. 1, pp. 98-102, 2017.

[14] G. Zhang, Q. Wang, M. Yang et al., "OSpaad: an online tool to perform survival analysis by integrating gene expression profiling and long-term follow-up data of 1319 pancreatic carcinoma patients," Molecular Carcinogenesis, vol. 59, no. 3, pp. 304-310, 2020, doi. 10.1002/mc.23154.

[15] L. Xie, Y. Dang, J. Guo et al., "High KRT8 expression independently predicts poor prognosis for lung adenocarcinoma patients," Genes, vol. 10, no. 1, p. 36, 2019.

[16] D. Szklarczyk, A. Franceschini, S. Wyder et al., "STRING v10: protein-protein interaction networks, integrated over the tree of life," Nucleic Acids Research, vol. 43, no. 1, pp. 447-452, 2014.

[17] D. Warde-Farley, S. L. Donaldson, O. Comes et al., "The GeneMANIA prediction server: biological network integration for gene prioritization and predicting gene function," Nucleic Acids Research, vol. 38, no. suppl_2, pp. 214-220, 2010.

[18] G. Zhang, Q. Wang, M. Yang et al., "OSblca: a web server for investigating prognostic biomarkers of bladder cancer patients," Frontiers in Oncology, vol. 9, p. 466, 2019.

[19] L. Xie, Q. Wang, Y. Dang et al., "OSkirc: a web tool for identifying prognostic biomarkers in kidney renal clear cell carcinoma," Future Oncology, vol. 15, no. 27, pp. 3103-3110, 2019.

[20] H. Li, S.-R. Zhang, H.-X. Xu et al., "SRPX2 and RAB31 are effective prognostic biomarkers in pancreatic cancer," Journal of Cancer, vol. 10, no. 12, pp. 2670-2678, 2019.

[21] H. Chen, Y. Kong, Q. Yao et al., "Three hypomethylated genes were associated with poor overall survival in pancreatic cancer patients," Aging (Albany NY), vol. 11, no. 3, pp. 885-897, 2019.

[22] C. Pang, Y. Gu, Y. Ding et al., "Several genes involved in the JAK-STAT pathway may act as prognostic markers in pancreatic cancer identified by microarray data analysis," Medicine, vol. 97, no. 50, Article ID e13297, 2018.

[23] J. L. Ambrus, J. Pippin, A. Joseph et al., "Identification of a cDNA for a human high-molecular-weight B-cell growth factor," Proceedings of the National Academy of Sciences, vol. 90, no. 13, pp. 6330-6334, 1993.

[24] N. Leca, M. Laftavi, L. Shen, K. Matteson, J. Ambrus, and O. Pankewycz, "Regulation of human interleukin 14 transcription in vitro and in vivo after renal transplantation," Transplantation, vol. 86, no. 2, pp. 336-341, 2008.

[25] B. G. Bodine, B. G. Bennion, E. Leatham et al., "Conditionally induced RAGE expression by proximal airway epithelial cells in transgenic mice causes lung inflammation," Respiratory Research, vol. 15, no. 1, p. 133, 2014.

[26] E. Helen Kemp, R. A. Ajjan, R. A. Metcalfe, P. F. Watson, and A. P. Weetman, "IL-14 and IL-16 are expressed in the thyroid of patients with either Graves' disease or Hashimoto's thyroiditis," Clinical Endocrinology, vol. 83, no. 5, pp. 726-732, 2015.

[27] G. Xiao, Y. Cao, X. Qiu, W. Wang, and Y. Wang, "Influence of gender and age on the survival of patients with nasopharyngeal carcinoma," BMC Cancer, vol. 13, no. 1, p. 226, 2013.

[28] A. Micheli, A. Mariotto, A. Giorgi Rossi, G. Gatta, and P. Muti, "The prognostic role of gender in survival of adult cancer patients," European Journal of Cancer, vol. 34, no. 14, pp. 2271-2278, 1998.

[29] A. W. M. Lee, W. M. Sze, J. S. K. Au et al., "Treatment results for nasopharyngeal carcinoma in the modern era: the Hong Kong experience," International Journal of Radiation Oncology*Biolog $y *$ Physics, vol. 61, no. 4, pp. 1107-1116, 2005. 